\title{
Convergence of a heat flow on a Hilbert manifold
}

\author{
Piotr Rybka \\ Institute of Applied Mathematics, Warsaw University \\ ul.Banacha 2, 02-097 Warsaw, POLAND \\ rybka@mimuw.edu.pl \\ September 20, 2004
}

\begin{abstract}
We study the heat flow projected on a manifold $\mathcal{M} \subset L^{2}(\Omega)$. This manifold is defined by the condition that the integrals $\int_{\Omega} u^{k}(t, x) d x, k=1, \ldots, N$ are constants of motion. We show that solutions to this problem converge to a steady state as time goes to $+\infty$. We use in an essential way a variant of the Łojasiewicz inequality.
\end{abstract}

\section{Introduction}

It is well-known that the heat equation

$$
u_{t}=\Delta u
$$

generates a gradient flow in $L^{2}(\Omega)$. We are interested here in behavior of this flow projected on $\mathcal{M}$ a subset of $L^{2}(\Omega)$,

$$
\mathcal{M}=\left\{u \in L^{2}(\Omega) ; \int_{\Omega} u^{k}(x) d x=C_{k}, \quad k=1, \ldots, N\right\} .
$$

We fix our attention on $\Omega$ which is bounded region in $\mathbb{R}^{2}$ with smooth boundary. Under natural conditions $\mathcal{M}$ is a Hilbert manifold. In fact we can consider any finite set of integers $\mathcal{I}$ containing 1 instead of $\{1, \ldots, N\}$ in the above definition.

The equation in question takes the form

$$
u_{t}=\Delta u-\sum_{k=1}^{N} \lambda_{k} u^{k-1} \quad \text { in } \quad \Omega .
$$

We augment it with Neumann boundary condition and initial data:

$$
\frac{\partial u}{\partial \mathbf{n}}=0 \quad \text { on } \quad \partial \Omega, \quad u(0, x)=u_{0}(x)
$$

where $\mathbf{n}$ is the outer normal to $\partial \Omega$. 
The coefficients $\lambda_{k}$, which are the Lagrange multipliers, are defined by the requirement $u_{t}$ is tangent to $\mathcal{M}$

or equivalently, that

$$
I_{k}(u) \equiv \int_{\Omega} u^{k}(x) d x, \quad k=1, \ldots, N
$$

are constants of motion, we set here $I_{1}=0$. These conditions mean that $\Delta u-\sum_{k=1}^{N} \lambda_{k} u^{k-1}$ has to be orthogonal in $L^{2}(\Omega)$ to gradients of $u^{k}$, i.e. to $u^{k-1}$.

In Proposition 2.1 below we show that in cases of our interest it is easy to guarantee that the vectors $1, u, \ldots, u^{N-1}$ are linearly independent. Then, $\mathcal{M}$ is indeed a Hilbert manifold.

In this paper we show that there exists a unique solution to (1.1)-(1.2) and this solution has the following properties:

(i) the orbit of any $u_{0} \in W^{2, p}(\Omega)$ is precompact in $W^{2, p}(\Omega)$, for some $p>2$.

(ii) any solution $u$ has a $\operatorname{limit}_{t \rightarrow \infty} u(t)=u^{\infty}$ in $W^{2, p}(\Omega)$.

At this point let us recall a well-known result of L.Simon, see [6]. His work implies that solutions of the non-linear heat equations

$$
u_{t}=\Delta u-f(x, u)
$$

in $\Omega \subset \mathbb{R}^{n}$ which are bounded in $C^{2, \mu}$ have limits as $t \rightarrow \infty$. This result depends crucially on analyticity of the nonlinearity. We remark here that much weaker assumptions are sufficient if $n=1$, see the papers [7], [4] and [1].

The point of assuming analyticity of the source term $f$ in (1.3) is that it implies the Łojasiewicz inequality. Namely, we define a functional $E$ on $W^{1,2}(\Omega)$ by formula

$$
E(u)=\int_{\Omega}\left[\frac{1}{2}|\nabla u|^{2}+F(x, u)\right] d x,
$$

where $\frac{\partial F}{\partial u}=f$. If $v_{0}$ is a critical point of $E$, i.e. $v_{0}$ is a stationary point of (1.3) and $f$ is analytic, then for some $\vartheta \in\left(\frac{1}{2}, 1\right)$

$$
\left|E(v)-E\left(v_{0}\right)\right|^{1-\vartheta}<\|\nabla E(v)\|_{L^{2}}
$$

for all $v$ satisfying $\left\|v-v_{0}\right\|_{X}<\beta$. The point is to choose the right function space $X$. While Simon proved (1.4) for $X=C^{2, \mu}, \mu>0$ we shall use a version of his result established for $X=W^{2, p}$ by Jendoubi, (see [3, Proposition 1.3] and Proposition 3.1 below). There is also a substantial and growing body of recent literature on variants of generalization of the Łojasiewicz inequality. However, our research goes in different direction and we shall not comment on results of other authors.

One is tempted here to use Simon's type of argument or its simplified version (e.g. see [5], [3]) directly. However, this is not possible simply because our flow is on a manifold $\mathcal{M} \subset L^{2}$, and the gradient structure of (1.1)-(1.2) has to established yet. But first of all we have to clarify the issue of existence, the smoothness and finally the already mentioned boundedness of solutions. We deal with these tasks in $\S 2$. The proof of boundedness of $u(t)$ uses the homogeneity of $\lambda_{k}(u)$, which seems interesting for its own sake.

The main convergence result is shown in $\S 3$. It is obtained by selecting a proper functional $\mathcal{E}$ making the flow (1.1)-(1.2) a gradient flow of $\mathcal{E}$. Next we use an argument based on Łojasiewicz inequality as explained in [5]. 


\section{The heat flow on $\mathcal{M}$ and the bounds on $u$}

We shall establish existence of solutions to

$$
\left\{\begin{array}{l}
u_{t}=\Delta u-\sum_{k=1}^{N} \lambda_{k} u^{k-1} \quad \text { in } \Omega \subset \mathbb{R}^{2} \\
\frac{\partial u}{\partial u}=0 \text { on } \partial \Omega, \quad u(0, x)=u_{0},
\end{array}\right.
$$

where $\Omega$ is a bounded region with smooth boundary and $\lambda_{k}=\lambda_{k}(u)$ are such that $u_{t}$ is orthogonal to $\operatorname{span}\left\{u^{k-1}\right\}$ :

$$
u_{t} \perp \operatorname{span}\left\{u^{k-1}: k=1, \ldots, N\right\} .
$$

As a result, the flow will conserve the quantities

$$
I_{k}(u)=\int_{\Omega} u^{k}(x) d x \quad k=1, \ldots, N .
$$

We note that the condition (2.2) leads to the following set of equations. Namely, once we integrate by parts the result of multiplication of (2.1) by $u^{i-1}, i=1, \ldots, N$ we obtain

$$
0=\int_{\Omega} u^{i-1} u_{t}=\int_{\Omega}\left(\Delta u-\sum_{k=1}^{N} \lambda_{k} u^{k-1}\right) u^{i-1} d x, \quad i \geq 1
$$

i.e. we have

$$
-(i-1) \int_{\Omega}|\nabla u|^{2} u^{i-2} d x=\sum_{k=1}^{N} \lambda_{k} \int_{\Omega} u^{k+i-2} d x .
$$

If $i=1$, then we see

$$
0=\sum_{k=1}^{N} \lambda_{k} \int_{\Omega} u^{k-1} d x \quad \text { i.e. } \quad \frac{d}{d t} \int_{\Omega} u d x=0
$$

because we have chosen $\int_{\Omega} u_{0} d x=0$.

Equations (2.3) can be rewritten as

$$
\sum_{j=1}^{N} G_{i j}(u) \lambda_{j}=-(i-1) E_{i}, \quad i=1, \ldots, N
$$

where

$$
E_{1} \equiv 0, \quad E_{i}(u)=\int_{\Omega}|\nabla u|^{2} u^{i-2} d x, \quad \text { for } i \geq 2
$$

and

$$
G_{i j}(u)=\int_{\Omega} u^{i+j-2} d x
$$

We note $G=\left(G_{i j}\right)$ is the Gramm matrix of vectors $1, u, u^{2}, \ldots, u^{N-1}$ in $L^{2}(\Omega)$. Thus, (2.4) has a unique solution provided that these vectors are linearly independent. Once we know it, then due to Cramer's formula we obtain

$$
\lambda_{k}=-\operatorname{det} A_{k} / \operatorname{det} G, \quad k=1, \ldots, N,
$$


where matrix $A_{k}, k=1, \ldots, N$ is obtained from $G$ by replacing its $k$-th column with a vector

$$
\vec{E}=\left(0, E_{2}, \ldots,(N-1) E_{N}\right)^{T} \text {. }
$$

It turns out that it is quite easy to guarantee linear independence of $\left\{1, u, \ldots, u^{N-1}\right\}$, for we have:

Proposition 2.1 Let us suppose that $u \in C(\bar{\Omega}), \int_{\Omega} u(x) d x=0$ and $\int_{\Omega} u^{2}(x) d x>0$. Then the vectors $1, u, u^{2}, \ldots, u^{N-1}$ are linearly independent.

Proof. The above conditions imply that there exist $x_{i} \in \Omega, i=1, \ldots, N$ such that

$$
u\left(x_{i}\right) \neq u\left(x_{j}\right) \neq 1 \quad i \neq j .
$$

Thus the condition

$$
\sum_{k=1}^{N} \mu_{k} u^{k-1}=0, \quad i \neq j,
$$

evaluated at $x_{i}$ gives a linear system for $\mu_{k}$ 's. Its determinant is the Vandermonde determinant. It is never zero if (2.6) holds. The Proposition follows.

Once we defined $\lambda_{k}$ 's we may state an existence result.

Proposition 2.2 Let us suppose that $\Omega \subset \mathbb{R}^{2}$ is an open, bounded region with smooth boundary. We assume that $\lambda_{k}$ 's are defined by (2.5). Then equation (2.1) has a unique solution $u \in$ $C\left([0, T], W^{2,2}\right) \cap C^{1}\left((0, T), L^{2}\right)$ provided that $u_{0} \in W^{2,2}(\Omega), \frac{\partial u_{0}}{\partial \mathbf{n}}=0$ and $\int_{\Omega} u_{0}(x) d x=0$.

Proof. To some extent this is a standard application of the semigroup theory as in the book of Henry, see [2], with correction in the Russian translation. Namely, we know that $-\Delta$ : $D(-\Delta) \subset L^{2}(\Omega) \rightarrow L^{2}(\Omega)$ is a sectorial operator, where

$$
D(-\Delta)=\left\{u \in W^{2,2}(\Omega): \frac{\partial u}{\partial \mathbf{n}}=0, \int_{\Omega} u d x=0\right\}
$$

Moreover, due to the embedding theorems (see [2, Theorem 1.6.1]) and the algebraic structure of $\lambda_{k}$, (see (2.5)) the mappings

$$
X^{\alpha} \ni u \rightarrow \lambda_{k} u^{k-1} \in L^{2}(\Omega), \quad k=1, \ldots, N
$$

are locally Lipschitz continuous for any $\alpha \in\left(\frac{1}{2}, 1\right)$, where $X^{\alpha} \equiv\left(L^{2}\right)^{\alpha}$ denotes the fractional power of $L^{2}$, (see [2] for details). Thus, by [2, Theorem 3.3.3] there exists a unique solution $u$ to $(2.1)$ such that

$$
\left.u \in C\left([0, T), X^{\alpha}\right) \cap C\left((0, T), W^{2,2}\right) \cap C^{1}(0, T), L^{2}\right) .
$$

Next in order to show the desired smoothness we study the variation of constants formula

$$
u(t)=e^{\Delta t} u_{0}+\int_{0}^{t} e^{\Delta(t-s)} \sum_{k=1}^{N} \lambda_{k} u^{k-1}(s) d s .
$$


The proof that $u \in C\left([0, T], W^{2,2}\right)$ requires a standard argument and it is left to the interested reader.

We may now check that indeed $I_{k}$ are constants of motion.

Proposition 2.3 Let us suppose that $u \in C\left([0, T), W^{2,2}\right)$ is a unique solution to (2.1). Then

$$
\frac{d}{d t} \int_{\Omega} u^{k} d x=0, \quad k=1, \ldots, N
$$

Proof. We may compute $\frac{d}{d t} \int_{\Omega} u^{k}$. We see

$$
\begin{aligned}
\frac{d}{d t} \int_{\Omega} u^{k} d x & =k \int_{\Omega} u_{t} u^{k-1} d x \\
& =k \int_{\Omega}\left(\Delta u-\sum_{j=1}^{N} \lambda_{j} u^{j-1}\right) u^{k-1} d x \\
& =-k(k-1) \int_{\Omega}|\nabla u|^{2} u^{k-2} d x-k \int_{\Omega} \sum_{j=1}^{N} \lambda_{j} u^{j+k-2} d x
\end{aligned}
$$

due to integration by parts utilizing the boundary conditions. Now the RHS vanishes because of the definition of $\lambda_{k}$, i.e. formula (2.5).

Thus, we established local in time existence of the flow of (2.1) on manifold $\mathcal{M}$ in other words, the solutions to (2.1) conserves the integrals

$$
\int_{\Omega} u^{k}(t, x) d x, \quad k=1, \ldots, N .
$$

Our next task is to make sure that the flow is globally defined, which is not obvious for nonlinear equations and to show enough bounds which guarantee precompactness of any orbit. For that purpose we have to understand better the non-linear term

$$
P(u)=\sum_{k=1}^{N} \lambda_{k}(u) u^{k-1} .
$$

Our subsequent analysis is based on homogeneity of $P$. Namely, we note:

Proposition 2.4 Suppose that $t>0$ and $1, u, \ldots, u^{N-1}$ are linearly independent, then

$$
\lambda_{k}(t u)=t^{2-k} \lambda_{k}(u) .
$$

Proof. The coefficients $\lambda_{u}$ 's are defined by (2.5). Hence it is sufficient to study homogeneity of $\operatorname{det} G(u)$ and $\operatorname{det} A_{k}(u)$. By inspection we see

$$
\operatorname{det} G(t u)=t^{(N-1) N} \operatorname{det} G(u)
$$

and

$$
\operatorname{det} A_{k}(t u)=t^{N(N-1)-k+2} \operatorname{det} t_{k}(u)
$$


and the desired result follows.

Let us notice that $\lambda_{k}$ 's are well-defined for $u \in X^{\alpha}, \alpha>\frac{1}{2}$, hence the quantity

$$
M_{\alpha}=\sup _{\|u\|=1}\|P(u)\|_{L^{2}}
$$

for $P$ defined above is also well-defined and finite. For, if $u_{n} \in X^{\alpha},\left\|u_{n}\right\|_{X^{\alpha}}=1$ were such a sequence that

$$
\left\|P\left(u_{n}\right)\right\|_{L^{2}} \rightarrow \infty
$$

when $n \rightarrow \infty$, then by compactness of the embedding $X^{\alpha} \subset X^{\alpha^{\prime}}, \alpha^{\prime}<\alpha$, there would be a subsequence $u_{n_{k}}$ converging to $u^{\infty}$ in $X^{\alpha^{\prime}}$. Due to continuity of

$$
X^{\beta} \ni u \rightarrow\|P(u)\|_{L^{2}} \in \mathbb{R}
$$

for $\beta>\frac{1}{2}$ we deduce

$$
\lim _{k \rightarrow \infty}\left\|P\left(u_{n_{k}}\right)\right\|_{L^{2}}=\left\|P\left(u^{\infty}\right)\right\|_{L^{2}} .
$$

This contradicts (2.8) and the claim follows.

We may now establish bounds on solutions implying that: (1) the solutions are global in time; (2) the orbit is precompact in $X^{\alpha}$ for sufficiently large $\alpha$, hence they are precompact in $W^{2, p}$, for some $p>2$.

Theorem 2.5 We assume that $u_{0} \in D(-\Delta)$, and $1, u_{0}, u_{0}^{2}, \ldots, u_{0}^{N-1}$ are linearly independent. Then, the unique solution $u$ to equation (2.1) is defined globally and it satisfies for any $\alpha \in\left[\frac{1}{2}, 1\right)$ the following estimate:

$$
\sup _{t>0}\|u(t)\|_{\alpha} \leq N_{\alpha}<\infty .
$$

Proof. The case $\alpha=\frac{1}{2}$ is elementary, because the norms $\|u\|_{X^{1 / 2}}$ and $\|\nabla u\|_{L^{2}}$ are equivalent. Hence, it is sufficient to check that

$$
\frac{d}{d t} \int_{\Omega} \frac{1}{2}|\nabla u|^{2} d x \leq 0
$$

Indeed, after integration by parts we arrive at

$$
\begin{aligned}
\frac{d}{d t} \frac{1}{2} \int_{\Omega}|\nabla u|^{2} d x & =\int_{\Omega} \nabla u \nabla u_{t} d x=\int_{\Omega}-\Delta u u_{t} d x \\
& =\int_{\Omega}\left(-\Delta u+\sum_{k=1}^{N} \lambda_{k} u^{k-1}\right) u_{t} d x=-\int_{\Omega}\left|u_{t}\right|^{2} d x \leq 0 .
\end{aligned}
$$

Here, in the above calculations we exploit the fact that $I_{k}$ are constants of motion.

Let us now take $\alpha \in\left(\frac{1}{2}, 1\right)$. The constant variation formula (2.7) implies

$$
(-\Delta)^{\alpha} u(t)=(-\Delta)^{\alpha} e^{\Delta t} u_{0}+\int_{0}^{t}(-\Delta)^{\alpha} e^{\Delta(t-s)} P(u(s)) d s .
$$

Due to [2, Theorem 1.4.3] we see

$$
\|u(t)\|_{X^{\alpha}} \leq e^{-\lambda t}\left\|u_{0}\right\|_{X^{\alpha}}+\int_{0}^{t} c_{\alpha} \frac{e^{-\lambda(t-s)}}{(t-s)^{\alpha}}\|P(u(s))\|_{L^{2}} d s .
$$


Note. Here $\lambda$ is the smallest eigenvalue of $-\Delta$ and $\lambda>0$, because no non-zero constant is allowed in $D(-\Delta)$. Moreover Proposition 2.4 implies,

$$
\begin{aligned}
P(u(s)) & =P\left(\frac{u(s)}{\tau} \tau\right)=\sum_{k=1}^{N} \lambda_{k}\left(\frac{u(s)}{\tau} \tau\right)\left(\frac{u(s)}{\tau}\right)^{k-1} \tau^{k-1} \\
& =P\left(\frac{u(s)}{\tau}\right) \tau .
\end{aligned}
$$

We shall take $\tau=\|u(s)\|_{X^{\alpha^{\prime}}}$. Thus, for any $\alpha^{\prime}$ satisfying $\frac{1}{2}<\alpha^{\prime}<\alpha$ we have the following string of estimates

$$
\begin{aligned}
\|u(t)\|_{X^{\alpha}} & \leq e^{-\lambda t}\left\|u_{0}\right\|_{X^{\alpha}}+c_{\alpha} \int_{0}^{t} \frac{e^{-\lambda(t-s)}}{(t-s)^{\alpha}} \sup _{0 \leq s \leq t}\|P(u(s))\|_{L^{2}} d s \\
& \leq\left\|u_{0}\right\|_{X^{\alpha}}+c_{\alpha} \int_{0}^{t} \frac{e^{-\lambda(t-s)}}{(t-s)^{\alpha}} \sup _{0 \leq s \leq t}\left\|P\left(\frac{u(s)}{\|u(s)\|_{X^{\alpha^{\prime}}}}\right)\right\|_{L^{2}}\|u(s)\|_{X^{\alpha^{\prime}}} d s \\
& \leq\left\|u_{0}\right\|_{X^{\alpha}}+c_{\alpha} \int_{0}^{t} \frac{e^{-\lambda(t-s)}}{(t-s)^{\alpha}} M_{\alpha^{\prime}}\|u(s)\|_{X^{\alpha^{\prime}}} d s .
\end{aligned}
$$

Inequality [2, Theorem 1.4.4] reads for $1>\beta>0$

$$
\left\|(-\Delta)^{\beta} x\right\| \leq \varepsilon\|(-\Delta) x\|+c \varepsilon^{\frac{-\beta}{1-\beta}}\|x\|
$$

for $x \in D(-\Delta)$. If $x=(-\Delta)^{\alpha-1} y$ and $\beta=1+\alpha^{\prime}-\alpha$, where $\alpha^{\prime}<\alpha$, then we obtain

$$
\begin{aligned}
\|y\|_{X^{\alpha^{\prime}}} & =\left\|(-\Delta)^{1+\alpha^{\prime}-\alpha}(-\Delta)^{\alpha-1} y\right\|_{L^{2}} \\
& \leq \varepsilon\left\|(-\Delta)(-\Delta)^{\alpha-1} y\right\|_{L^{2}}+c(\varepsilon)\left\|(-\Delta)^{\alpha-1} y\right\|_{L^{2} .}
\end{aligned}
$$

In other words we get

$$
\|y\|_{X^{\alpha^{\prime}}} \leq \varepsilon\|y\|_{X^{\alpha}}+C(\varepsilon)\|y\|
$$

because $A^{\alpha-1}$ is a continuous operator for $\alpha<1$.

Inequality (2.9) implies further estimates

$$
\|u\|_{X^{\alpha}} \leq e^{-\lambda t}\left\|u_{0}\right\|_{X^{\alpha}}+c_{\alpha} \int_{0}^{t} \frac{e^{-\lambda(t-s)}}{(t-s)^{\alpha}} M_{\alpha^{\prime}}\left(\varepsilon\|u(s)\|_{X^{\alpha}}+c(\varepsilon)\|u\|_{L^{2}}\right) d s
$$

We remark here, that $\|u\|_{L^{2}}^{2}=I_{2}$ is a constant of motion, however if $k=2$ is not in $\mathcal{I}$ we use Poincaré's inequality,

$$
\|u\|_{L^{2}} \leq\left\|u-\frac{1}{|\Omega|} \int_{\Omega} u\right\|_{L^{2}} \leq\|\nabla u\|_{L^{2}} \leq\left\|\nabla u_{0}\right\|_{L^{2}}
$$

In any case, we see

$$
\sup _{0 \leq s \leq t}\|u(s)\|_{X^{\alpha}} \leq e^{-\alpha}\left\|u_{0}\right\|_{X^{\alpha}}+\int_{0}^{t}\left(\varepsilon c_{\alpha} M_{\alpha} \frac{e^{-\alpha(t-s)}}{(t-s)^{\alpha}} \sup _{0 \leq s \leq t}\|u(s)\|_{X^{\alpha}}+c(\varepsilon) \sqrt{I_{2}}\right) d s
$$


We may now choose $\varepsilon$ such that

$$
\varepsilon c_{\alpha} M_{\alpha^{\prime}} \int_{0}^{t} \frac{e^{-\lambda(t-s)}}{(t-s)^{\alpha}} d s \leq \varepsilon \lambda^{\alpha-1} c_{\alpha} M_{\alpha^{\prime}} \int_{0}^{\infty} \frac{e^{-s}}{s^{\alpha}} d s=\varepsilon \lambda^{\alpha-1} c_{\alpha} M_{\alpha^{\prime}} \Gamma(1-\alpha)<\frac{1}{2} .
$$

Thus

$$
\begin{aligned}
\frac{1}{2} \sup _{s \leq t}\|u(s)\|_{X^{\alpha}} & \leq e^{-\lambda t}\|u\|_{X^{\alpha}}+\int_{0}^{t} c_{\alpha} M_{\alpha} \frac{e^{-\lambda(t-s)}}{(t-s)^{\alpha}} c(\varepsilon) \sqrt{I_{2}} \\
& =e^{-\lambda t}\left\|u_{0}\right\|_{X^{\alpha}}+c_{\alpha} M_{\alpha} \lambda^{\alpha-1} \Gamma(1-\alpha) \sqrt{I_{2}} c(\varepsilon)=N_{\alpha} .
\end{aligned}
$$

Now, we shall prove more:

Theorem 2.6 Let us suppose that $\beta \in\left(1,1 \frac{1}{2}\right)$, then

$$
\sup _{t \geq 1}\|u\|_{\beta} \leq S_{\beta}
$$

Proof. We use again the constant variation formula (2.7) and apply $(-\Delta)^{\beta}$ to its both sides.

This yields

$$
\begin{aligned}
\|u(t)\|_{X^{\beta}} & \leq t^{-\beta+1} e^{-\lambda t}\left\|\Delta u_{0}\right\|_{L^{2}}+C\left\|\int_{0}^{t}(-\Delta)^{\beta-\frac{1}{2}} e^{\Delta(t-s)} \nabla P(u)\right\|_{L^{2}} d s \\
& \leq t^{-\beta+1} e^{-\lambda t}\left\|\Delta u_{0}\right\|_{L^{2}}+\int_{0}^{t} \frac{c_{\alpha} e^{-\lambda(t-s)}}{(t-s)^{\frac{1}{2}-\beta}}\left\|\sum_{k=1}^{N} \lambda_{k}(k-1) u^{k-2} \nabla u\right\|_{L^{2}} d s,
\end{aligned}
$$

but $\left\|\sum_{k=1}^{N} \lambda_{k}(k-1) u^{k-2} \nabla u\right\|_{L^{2}} \leq K_{\alpha}<\infty$ due to Theorem 2.5. Hence the desired bound follows.

Corollary 2.7 There exist $p>2$ such that the orbit $\{u(t): t \geq 1\}$ is precompact in $W^{2, p}(\Omega)$.

Proof. We have just proved that $\{u(t): t \geq 1\}$ is bounded in $X^{\beta}$. Due to [2, Theorem 1.4.8] embedding $X^{\beta} \subset X^{\beta^{\prime}}$ is compact provided that $\beta^{\prime}<\beta$. Moreover, by [2, Theorem 1.6.1], if $\beta^{\prime}>1$, then $X^{\beta^{\prime}} \subset W^{2, p}$ for some $p>2$.

We may now state

Corollary 2.8 The set $\omega\left(u_{0}\right)$ exists and it is compact in $W^{2, p}$.

Proof. This follows from precompactness of $\{u(t): t \geq 1\}$ in $W^{2, p}$ by a general argument, see [2, Theorem 4.3.3]. 


\section{Convergence}

Once we settled the question of existence of dynamics and a compact $\omega$-limit set, we may turn to the main question which is convergence. We will use an argument based on analyticity of the non-linear term. More specifically we will use an infinite dimensional version of Łojasiewicz inequality due to Jendoubi:

Proposition 3.1 ([3, Proposition 1.3]) Let us suppose that $p>2$ and $\Omega \subset \mathbb{R}^{2}$ is a bounded region with smooth boundary. We assume that $\mathcal{E}: W^{2, p}(\Omega) \rightarrow \mathbb{R}$ is a functional of the form $\mathcal{E}(u)=\int_{\Omega}\left[\frac{1}{2}|\nabla u|^{2}+F(x, u)\right] d x$ where $F(x, u)$ uniformly in $x$ real analytic in $u$ and $u_{0}$ is its critical point. Then, there exist $\beta>0$ and $\vartheta \in\left(\frac{1}{2}, 1\right)$ such that

$$
\|\nabla \mathcal{E}(u)\|_{L^{2}} \geq\left|\mathcal{E}(u)-\mathcal{E}\left(u_{0}\right)\right|^{1-\vartheta}
$$

for all $u$ such that $\left\|u-u_{0}\right\|_{W^{2, p}}<\beta$.

Here is the main result of this paper.

Theorem 3.2 Let us suppose that $u_{0} \in W^{2,2}(\Omega), \int_{\Omega} u_{0}(x) d x=0, \frac{\partial u_{0}}{\partial \mathbf{n}}=0$ and $u_{0} \neq 0$. Then, the unique solution to (2.1) with this initial data has a limit $u^{\infty}$ as time goes to $+\infty$ in the norm of $W^{2, p}(\Omega)$, for some $p>2$.

Proof. We need to adapt the result of Proposition 3.1 to the case of our flow on a manifold. We shall set

$$
\mathcal{E}(u)=\frac{1}{2} \int_{\Omega}|\nabla u|^{2}+\sum_{k=1}^{N} \frac{\lambda_{k}}{k} \int_{\Omega}\left(u^{k}-\frac{C_{k}}{|\Omega|}\right),
$$

where constants $C_{k}, k=1, \ldots, N$ appear in the definition on $\mathcal{M}$. We notice that $\mathcal{E}(u)$ is well-defined on $W^{2,2}$ and coincides with $E(u)=\frac{1}{2} \int_{\Omega}|\nabla u|^{2}$ on $\mathcal{M}$.

If $w$ is a stationary point of (2.1) and $h$ any test function in $W^{1,2}$ with $\int_{\Omega} h d x=0$, then

$$
\begin{aligned}
\nabla \mathcal{E}(w) h & =\int_{\Omega} \nabla w \nabla h+\sum_{k=1}^{N} \frac{1}{k} \frac{\delta \lambda_{k}}{\delta u} \int_{\Omega}\left(w^{k}-\frac{C_{k}}{|\Omega|}\right)+\sum_{k=1}^{N} \lambda_{k} \int_{\Omega} w^{k-1} h \\
& =\int_{\Omega}-\Delta w h+\sum_{k=1}^{N} \lambda_{k} w^{k-1} h \\
& =0,
\end{aligned}
$$

i.e. $w$ is also a critical point of $\mathcal{E}$. Moreover, if $v \in W^{2, p}$ is a critical point of $\mathcal{E}$ and $I_{k}(v)=C_{k}$, then it is easy to see that $v$ is a stationary point of (2.1).

Let us calculate $\frac{d \mathcal{E}}{d t}$ along the orbit

$$
\begin{aligned}
\frac{d \mathcal{E}}{d t} & =\int_{\Omega} \nabla u \nabla u_{t}+\sum_{k=1}^{N} \frac{d \lambda_{k}}{d t} \frac{1}{k} \int_{\Omega}\left(u^{k}-\frac{C_{k}}{|\Omega|}\right) d x+\sum_{k=1}^{N} \lambda_{k} \int_{\Omega} u^{k-1} u_{t} d x \\
& =-\int_{\Omega} \Delta u u_{t}+\sum_{k=1}^{N} \lambda_{k} \int_{\Omega} u^{k-1} u_{t} d x=-\int_{\Omega} u_{t}^{2} d x \leq 0 .
\end{aligned}
$$


Once we notice that $\nabla \mathcal{E}$ evaluated at $\mathcal{M}$ coincides with $-\Delta u+\sum_{k=1}^{N} \lambda_{k} u^{k-1}$ we conclude the

$$
\frac{d \mathcal{E}}{d t}=-\|\nabla \mathcal{E}\|_{L^{2}}\left\|u_{t}\right\|_{L^{2}}
$$

Hence

$$
-\frac{d \mathcal{E}^{\vartheta}}{d t}=\frac{\vartheta\|\nabla \mathcal{E}\|_{L^{2}}}{\mathcal{E}^{1-\vartheta}}\left\|u_{t}\right\|_{L^{2}}
$$

Starting from this point, in order to finish the proof we repeat the argument which appeared in the proof of [5, Theorem 3.1].

We also know due to a general argument in [2, Theorem 4.3.3] that

$$
\operatorname{dist}\left(u(t), \omega\left(u_{0}\right)\right) \rightarrow 0
$$

in the norm of $W^{2, p}$. We also notice that

$$
\left.\mathcal{E}\right|_{\omega\left(u_{0}\right)}=e_{0}=\text { const. }
$$

and all points in $\omega\left(u_{0}\right)$ are critical points of $\mathcal{E}$.

By Proposition 3.1 for each $x \in \omega\left(u_{0}\right)$ there exist $\beta_{0}(x), \theta(x) \in\left(0, \frac{1}{2}\right)$ such that

$$
\|\nabla \mathcal{E}(v)\|_{W^{2, p}} \geq|\mathcal{E}(v)-\mathcal{E}(x)|^{1-\theta(x)}
$$

for all $v$ such that $\|v-x\|_{W^{2, p}}<\beta(x)$. These balls form an open covering of $\omega\left(u_{0}\right)$, due to compactness of this set there exists a finite covering

$$
\mathcal{U}=\bigcup_{j=1}^{m} B_{W^{2, p}}\left(w_{j}, \beta_{0}\left(w_{j}\right)\right) \supset \omega\left(u_{0}\right) .
$$

Because of (3.3) there exists $t_{0}>0$ such that for all $t>t_{0} u(t) \in \mathcal{U}$. Let us also set $\theta=$ $\min \left\{\theta\left(w_{1}\right), \ldots, \theta\left(w_{m}\right)\right\}$. It is now clear that (3.4) implies that

$$
\|\nabla \mathcal{E}(v)\|_{W^{2, p}} \geq\left|E(v)-e_{0}\right|^{1-\theta} \quad \text { for all } v \text { in } \mathcal{U} .
$$

Hence, (3.2) implies

$$
-\frac{d \mathcal{E}}{d t} \geq \vartheta\left\|u_{t}\right\|
$$

and finally $\int_{t}^{s}\left\|u_{t}\right\| \leq-\mathcal{E}(u(s))^{\vartheta}+\mathcal{E}(u(t))^{\vartheta}$.

Thus we are able to conclude that $u(t)$ converges in $L^{2}$, when $t \rightarrow \infty$,

$$
\lim _{t \rightarrow \infty} u(t)=u^{\infty}
$$

However, due to (3.3) convergence is in $W^{2, p}$.

Acknowledgment. The author acknowledges discussions with Prof. H.Matano on the subject of this paper. This topic was also suggested by him. 


\section{References}

[1] X.-Y. Chen, H.Matano, Convergence, asymptotic periodicity and finite-point blow-up in one-dimensional semilinear heat equations, J. Diff. Equations, 78 (1989), 160-190.

[2] D.Henry, Geometric theory of semilinear parabolic equations. Lecture Notes in Mathematics, vol. 840, Springer, Berlin 1981. (Russian translation: Mir, Moscow 1985).

[3] M.A.Jendoubi, A simple unified approach to some convergence theorems of L.Simon, J. Funct. Anal., 153, (1998), 187-202.

[4] H.Matano, Convergence of solutions of one-dimensional semilinear heat equation, $J$. Math. Kyoto Univ., 18, (1978), 221-227.

[5] P.Rybka, K.-H.Hoffmann, Convergence of solutions to equation of quasi-static approximation of viscoelasticity with capillarity, J.Math. Anal. Appl., 226, (1998), 61-81.

[6] L.Simon, Asymptotics for a class of non-linear evolution equations, with applications to geometric problems, Ann. of Math., 118, (1983), 525-571.

[7] T.I.Zelenyak, Stabilization of solutions of boundary value problems for a second-order parabolic equation with one space variable, Differentsial'nye Uravneniya, 4, (1968), 1722. 